Article

\title{
Inkjet-Printed Molybdenum Disulfide and Nitrogen- Doped Graphene Active Layer High On/Off Ratio Transistors
}

\author{
Mohi Uddin Jewel ${ }^{1}$ (D), Mahmuda Akter Monne ${ }^{2} \mathbb{D}$, Bhagyashree Mishra ${ }^{2}$ and \\ Maggie Yihong Chen $1,2, *$ (D)
}

1 Ingram School of Engineering, Texas State University, San Marcos, TX 78666, USA; jewel.eee08@gmail.com

2 Materials Science, Engineering, and Commercialization, Texas State University, San Marcos, TX 78666, USA; mahmuda.a.monne@txstate.edu (M.A.M.); b_m415@txstate.edu (B.M.)

* Correspondence: Maggie.chen@txstate.edu; Tel.: +1-512-996-8778

Academic Editors: Xuejun Lu and Derek J. McPhee

Received: 24 December 2019; Accepted: 26 February 2020; Published: 28 February 2020

\begin{abstract}
Fully inkjet-printed device fabrication is a crucial goal to enable large-area printed electronics. The limited number of two-dimensional (2D) material inks, the bottom-gated structures, and the low current on/off ratio of thin-film transistors (TFTs) has impeded the practical applications of the printed 2D material TFTs. In the search for TFTs with high current ratios, we introduce a stable and efficient method of nitrogen-doped graphene (NDG) ink preparation for inkjet printing by liquid-phase exfoliation. The NDG thin film is print-stacked with molybdenum disulfide $\left(\mathrm{MoS}_{2}\right)$ by multiple printing passes to construct a $\mathrm{MoS}_{2}-\mathrm{NDG}$ stack. We demonstrate top-gated fully inkjet-printed $\mathrm{MoS}_{2}-\mathrm{NDG}$ transistors with silver drain, source, and gate electrodes, and a barium titanate $\left(\mathrm{BaTiO}_{3}\right)$ dielectric. A $100 \%$ inkjet-printed $\mathrm{MoS}_{2}-\mathrm{NDG}$ vertical 2D active heterostructure layer transistor with a current on/off ratio of 1200 is exhibited. The results may lead towards the development of all-printed 2D material-based transistor switches.
\end{abstract}

Keywords: inkjet printing; graphene; molybdenum disulfide; Raman; thin-films; cross-section; nanosheets; on/off ratio; transistor

\section{Introduction}

Graphene and related two-dimensional (2D) materials remain at the center of research for more than a decade because of their exciting and unusual properties [1]. Printing of solution-processed 2D materials facilitates the fabrication of the next generation of flexible devices. Inkjet printing as a process for flexible and printed electronics, has become more popular for the fabrication of electronic devices because of its ability for large-area fabrication, the limited number of process steps involved, low-temperature processing, and low cost [2]. Graphene is a zero-band gap semimetal in which the valence and conduction bands are cone shaped, and they meet at the K point of the Brillouin zone. Due to the absence of band gap, the typical current on/off ratio of pristine graphene thin-film transistors (TFTs) is approximately 5 [3]. For switching applications of TFTs, a current on/off ratio between $10^{4}$ and $10^{7}$ and a band gap of thin film larger than $0.36 \mathrm{eV}$ are desirable [4]. As with regular thin-film graphene TFTs, the inkjet-printed graphene transistors had current ratios of $\approx 10[2,5]$. Recently, all inkjet-printed graphene transistors were reported for wearable and textile electronics [6]. However, the current on/off ratio was still small (about 2.5). Molybdenum disulfide $\left(\mathrm{MoS}_{2}\right)$, a semiconducting layered transition metal dichalcogenide (TMD), is one of the most studied 2D materials after graphene. Mono and few-layered $\mathrm{MoS}_{2}$ have a direct band gap of $1.8 \mathrm{eV}$, while bulk $\mathrm{MoS}_{2}$ has an indirect band gap of $1.29 \mathrm{eV}$ [7]. Despite having a band gap in $\mathrm{MoS}_{2}$, liquid phase-exfoliated (LPE) and inkjet-printed $\mathrm{MoS}_{2}$ 
transistors had low current on/off ratios $(<10)$ [8-11]. A maximum current on/off ratio of 600 was demonstrated for the electrolytically gated TFTs based on porous nanosheet networks (PNNs) of 2D materials [12]. However, the TFTs were not fully printed. For example, the channel materials were spray coated, the source and drain electrodes were deposited by electron beam (e-beam) evaporation, and the liquid electrolyte was drop casted [12]. Yet, their reported all-printed TFTs with inkjet-printed graphene source, drain, and gate, and tungsten diselenide (WSe $\left.{ }_{2}\right)$ channel, a spray-coated porous boron nitride (BN) electrochemical separator (not a dielectric), and a drop-casted ionic liquid, displayed on/off ratios of $\approx 25$. Very recently, solution-processed $2 \mathrm{D}$ material back-gated TFTs with an on/off ratio of $>10^{5}$ were reported using standard photolithography process [13]. The devices available in the literature are mostly bottom gated and printed devices have low on/off ratios which are not suitable for practical application of a transistor switch. Therefore, it is indispensable to develop top-gated TFTs with high on/off ratios for printed transistor switches.

Several attempts have been made to open the band gap in graphene through graphene-substrate interaction, chemical substitution doping, and quantum confinement [14-16]. A band gap of $0.25 \mathrm{eV}$ was achieved for chemical vapor-deposited (CVD) nitrogen-doped graphene (NDG) nanosheets [15]. Because of the band gap opening in NDG, it has a great potential to be an excellent material for inkjet-printed transistors with high current ratios when coupled with semiconducting $\mathrm{MoS}_{2}$. In this work, we report the development of NDG and $\mathrm{MoS}_{2}$ inks, fabrication, and characterization of a fully printed top-gated $\mathrm{MoS}_{2}-\mathrm{NDG}$ stack field effect transistor. We designed a printed vertical $\mathrm{MoS}_{2}-\mathrm{NDG}$ stack for the transistor channel. The all-printed transistor displays a current on/off ratio of 1200 .

\section{Results}

\subsection{NDG and $\mathrm{MoS}_{2}$ Nanosheets}

The flake dimensions must match the requirements of drop-on-demand (DOD) printing. The lateral dimensions of the dispersed nanosheets should be at least $1 / 50$ of the nozzle diameter $(\mathrm{a} \approx 21.5 \mu \mathrm{m})$ to prevent the nozzle clogging. We purchased the NDG powder from Sigma-Aldrich (product no. 900527) and $\mathrm{MoS}_{2}$ ultrafine powder from Graphene supermarket. The scanning electron microscopy (SEM) images of bulk nanosheets are shown in Figure 1a-b. Prior to dispersing the nanosheets into solvents, the nanosheets were sonicated for $10 \mathrm{~h}$ to break the bulk nanosheets. The lateral size and thickness of the individual NDG and $\mathrm{MoS}_{2}$ nanosheets were extracted from atomic force microscopy (AFM) measurements for over 50 flakes as shown in Figure 1c-f. The flake size follows the normal distribution (peak $\approx 150 \mathrm{~nm}$ ) for NDG and log-normal distribution (peak $\approx 100 \mathrm{~nm}$ ) for $\mathrm{MoS}_{2}$, respectively, which fulfills the DOD requirements. The statistical thickness of normal distribution for NDG nanosheets peaked at $20 \mathrm{~nm}$, and the Lorentz distribution of thickness for $\mathrm{MoS}_{2}$ nanosheets peaked at $25 \mathrm{~nm}$, respectively. Assuming a thickness of $0.37 \mathrm{~nm}$ for monolayer graphene and $0.615 \mathrm{~nm}$ for monolayer $\mathrm{MoS}_{2}[17,18]$, the nanosheets consist of an average number of layers; $\mathrm{N} \approx 54$ for the NDG and $\mathrm{N} \approx 40$ for the $\mathrm{MoS}_{2}$. 

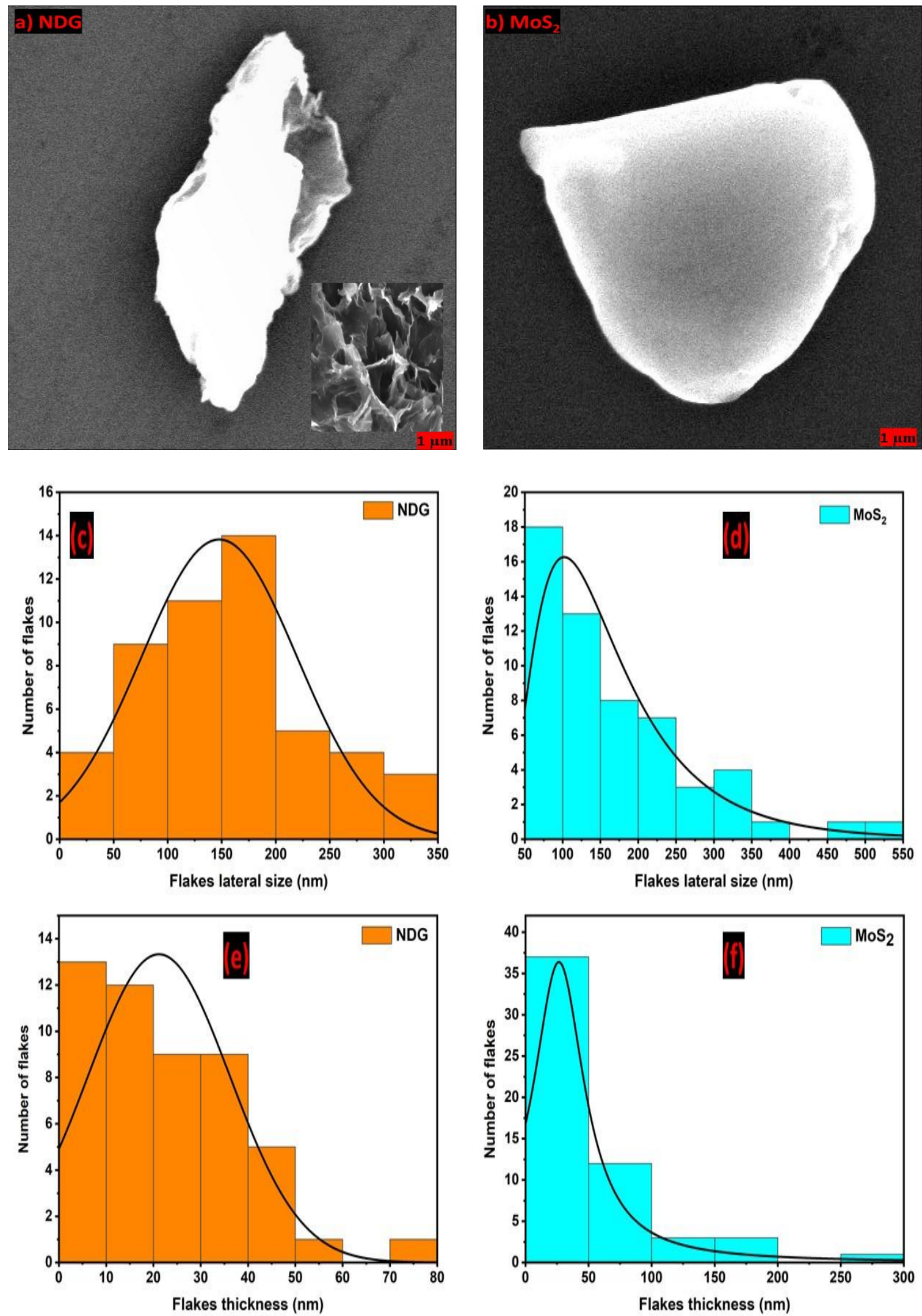

Figure 1. Scanning electron microscopy (SEM) images of (a) bulk nitrogen-doped graphene (NDG) nanosheets (inset: SEM image of broken NDG nanosheets), and (b) molybdenum disulfide $\left(\mathrm{MoS}_{2}\right)$ as-purchased flakes. Lateral size distribution from atomic force microscopy (AFM) measurements of (c) NDG and (d) $\mathrm{MoS}_{2}$ flakes. Flake thickness distribution of (e) NDG and (f) $\mathrm{MoS}_{2}$. 
Raman is one of the most common vibrational spectroscopies for fingerprinting carbon species. After the sonication, the Raman spectrum of NDG nanosheets on Si substrate was acquired under ambient conditions, which is shown in Figure 2a. The NDG shows two intense peaks, which are the $\mathrm{D}\left(\approx 1341 \mathrm{~cm}^{-1}\right)$ and $\mathrm{G}\left(\approx 1578 \mathrm{~cm}^{-1}\right)$ peaks, respectively. There are two additional peaks, $2 \mathrm{D}(\approx 2670$ $\left.\mathrm{cm}^{-1}\right)$ and $\mathrm{D}+\mathrm{D}^{\prime}\left(\approx 2904 \mathrm{~cm}^{-1}\right)$, present in the NDG nanosheets. The $\mathrm{G}$ and $2 \mathrm{D}$ peaks are the signature of graphene-like structure in nanosheets. The $\mathrm{G}$ band corresponds to $\mathrm{E}_{2 \mathrm{~g}}$ phonons at the Brillouin zone center. The $\mathrm{G}$ band arises from the stretching of $\mathrm{C}-\mathrm{C}$ bonds in a $\mathrm{sp}^{2}$ hybridized carbon system. The $\mathrm{D}$ band is related to defects in $\mathrm{sp}^{2}$ hybridized carbon materials, which is activated by an inter-valley double resonance (DR) Raman process. The intensity ratio $\mathrm{I}_{\mathrm{D}}$ (intensity of $\mathrm{D}$ peak)/ $\mathrm{I}_{\mathrm{G}}$ (intensity of $\mathrm{G}$ peak) of 1.005 indicates a high degree of disorder due to nitrogen doping. The strong $\mathrm{D}$ peak can also originate from the submicrometer flake boundaries and vacancies. The $2 \mathrm{D}$ peak is the secondary $\mathrm{D}$ peak, which appears at approximately $2700 \mathrm{~cm}^{-1}$. For single-layer graphene, the 2D band shows a sharp peak and high intensity compared to the D and G peaks [19]. The 2D band broadens and the intensity reduces with the number of graphene layers. In the Raman spectrum of Figure $2 a$, the intensity of the $2 \mathrm{D}$ band is much smaller compared to the $\mathrm{D}$ and $\mathrm{G}$ bands. We attribute the low intensity of the $2 \mathrm{D}$ band to the presence of many layers $(\mathrm{N} \approx 54)$ in NDG nanosheets. This Raman spectrum strongly agrees with the Raman spectra previously reported for NDG synthesized by chemical vapor deposition at 800 and $900{ }^{\circ} \mathrm{C}$ [20]. Figure $2 \mathrm{~b}$ shows the Raman spectrum of $\mathrm{MoS}_{2}$ flakes with characteristic $\mathrm{E}_{1 \mathrm{~g}}$ and $A_{1 g}$ peaks at 378 and $405 \mathrm{~cm}^{-1}$ respectively, which are obtained by Lorentzian fits in the spectrum.
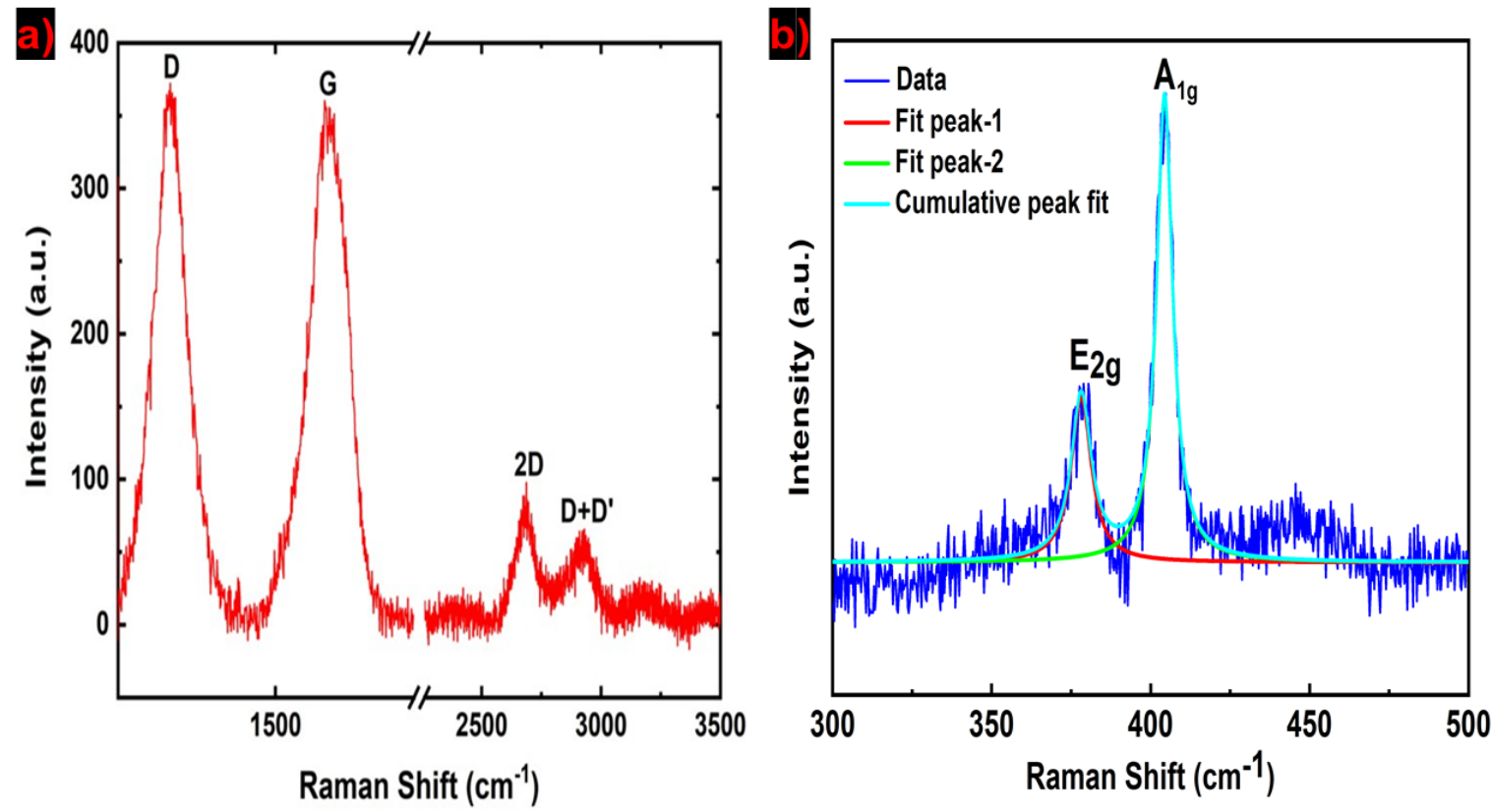

Figure 2. (a) Raman spectrum of NDG nanosheets; (b) Raman spectrum and their Lorentzian fits of $\mathrm{MoS}_{2}$ nanosheets.

\subsection{Printable Inks and Thin Films}

In this study, we used a commercial drop-on-demand (DOD) Fujifilm Dimatix materials printer (DMP-2800). For DOD inkjet printing, the material inks must have the ability to generate droplets. The droplets ejecting out from a nozzle are influenced by ink viscosity $\eta$ ( $\mathrm{mPa} s$ ), surface tension $\gamma(\mathrm{mN} / \mathrm{m})$, particle density $\rho\left(\mathrm{g} / \mathrm{cm}^{3}\right)$, and nozzle diameter a $(\mu \mathrm{m})$. We prepared and characterized the NDG and $\mathrm{MoS}_{2}$ inks (see 'Materials and Methods'). For inkjet printing of an ink, the inverse Ohnesorge number $\left(\mathrm{Z}=\mathrm{Oh}^{-1}\right)$ is used as the figure of merit (FOM) to characterize the drop formation and jettability of an ink. $Z$ is calculated by using $Z=\frac{(\gamma \rho)^{1 / 2}}{\eta}$ and an optimal range of $Z$ between 1 and 14 is required for stable DOD printing [5]. For NDG ink, using $\eta=2.94 \mathrm{mPa}, \gamma=20.98 \mathrm{mN} / \mathrm{m}$, 
$\rho=1.054 \mathrm{~g} / \mathrm{cm}^{-3}, \mathrm{a}=21.5 \mu \mathrm{m}$, we obtain $\mathrm{Z}=7.5$, which is within the conventional range for inkjet printing. There were no satellite drops following the primary drops, which was confirmed by capturing the dynamics of drop formation. The $\mathrm{Z}$ for $\mathrm{MoS}_{2}$ and barium titanate $\left(\mathrm{BaTiO}_{3}\right)$ inks were 2 and 3.4, which are suitable for inkjet printing.

The concentration of nanosheets in the ink can be calculated from the UV-vis absorbance spectrum via the Beer-Lambert law. The Beer-Lambert law can be expressed as $\mathrm{A}=\alpha \mathrm{cl}$, where $\mathrm{A}$ is the absorbance, $\alpha$ is the absorption coefficient $\left(\mathrm{Lg}^{-1} \mathrm{~m}^{-1}\right), \mathrm{c}$ is the concentration of dispersed graphene $(\mathrm{g} / \mathrm{L})$, and 1 is the light path length $(\mathrm{m})$. The optical absorption of the NDG ink is shown in Figure 3a. The spectrum is mostly featureless due to the linear dispersion of the Dirac electrons, whereas the peak in the UV region is a signature of Hove singularity in the graphene density of states [6]. From $\mathrm{A}=0.501$, $\alpha=2460 \mathrm{Lg}^{-1} \mathrm{~m}^{-1}, 1=0.01 \mathrm{~m}$, our estimated NDG concentration is $0.4 \mathrm{mg} / \mathrm{mL}$ [21]. Figure $3 \mathrm{~b}$ shows the optical absorbance spectra for as prepared $\mathrm{MoS}_{2}$ ink. This absorbance spectrum complies with the previously reported $\mathrm{MoS}_{2}$ ink absorbance spectra [8]. For $\mathrm{MoS}_{2}$ presence in dispersion, two characteristic peaks appear at approximately 600 and $672 \mathrm{~nm}$ wavelength respectively [10]. Two excitonic absorption peaks at 605 and $666 \mathrm{~nm}$ are observed, which arise from the K points of the Brillouin zone in $\mathrm{MoS}_{2}$ nanosheets. The peak at $666 \mathrm{~nm}$ corresponds to the lowest optical bandgap of $1.86 \mathrm{eV}$ for $\mathrm{MoS}_{2}$ nanosheets, which is higher than the bulk $\mathrm{MoS}_{2}$ bandgap of $1.3 \mathrm{eV}$ [22]. The extinction coefficient of $\mathrm{MoS}_{2}$ at $672 \mathrm{~nm}$ wavelength is $\sim \alpha_{672}=3400 \mathrm{~mL} /(\mathrm{mg} . \mathrm{m})$ [8]. Using the Beer-Lambert law, the calculated final $\mathrm{MoS}_{2}$ concentration is $\sim 0.1 \mathrm{mg} / \mathrm{mL}$.
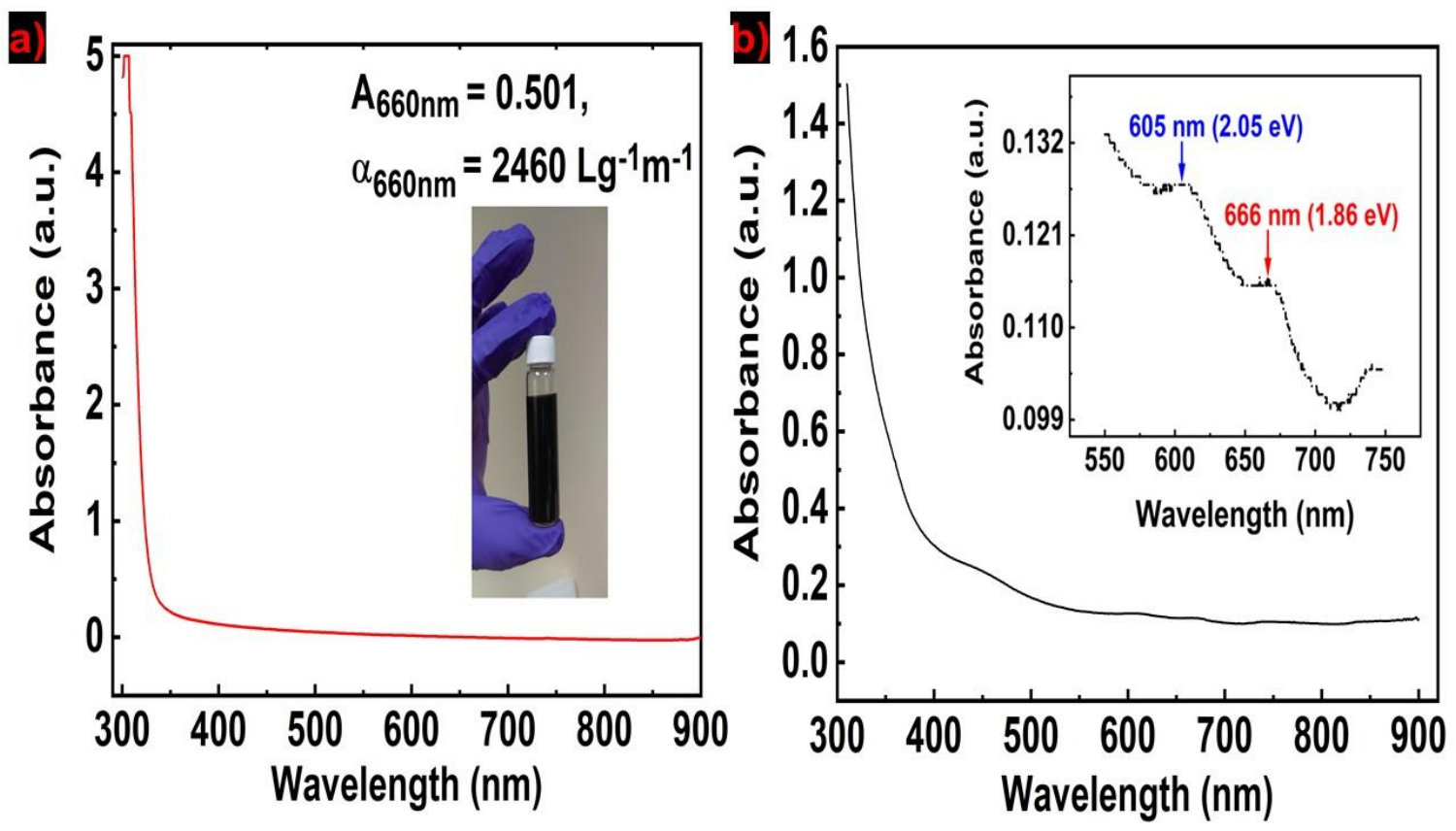

Figure 3. (a) Optical absorption of NDG ink shown in the inset; (b) absorbance spectra of $\mathrm{MoS}_{2}$ ink (inset: peak positions in $\mathrm{MoS}_{2}$ ink).

Figure 4 compares the Raman spectrum of the NDG powder and printed layers. As with NDG powder, the Raman spectra of the printed thin films have D, G, 2D, and D + D' peaks, respectively. The peaks of the Raman spectra remain identical with additional printing passes. The distribution of Raman bands in the printed layers remain similar to the NDG powder. The lower intensity of 2D peak indicates the presence of many layers of graphene in the printed thin films. Doping has strong effects on the position and FWHM of the 2D peak. The 2D peak position varied between 2667 and $2711 \mathrm{~cm}^{-1}$. We also calculated the full width at half maximum (FWHM) of the 2D peaks for the Raman spectra shown in Figure 4. The FWHM for the NDG powder, 2, 5 and 10 printed layers was 77.4, 63.6, 62.6, 
and 92.9, respectively. This, along with 2D peak position shift, implies that the nitrogen doping has a strong effect on printed NDG samples.

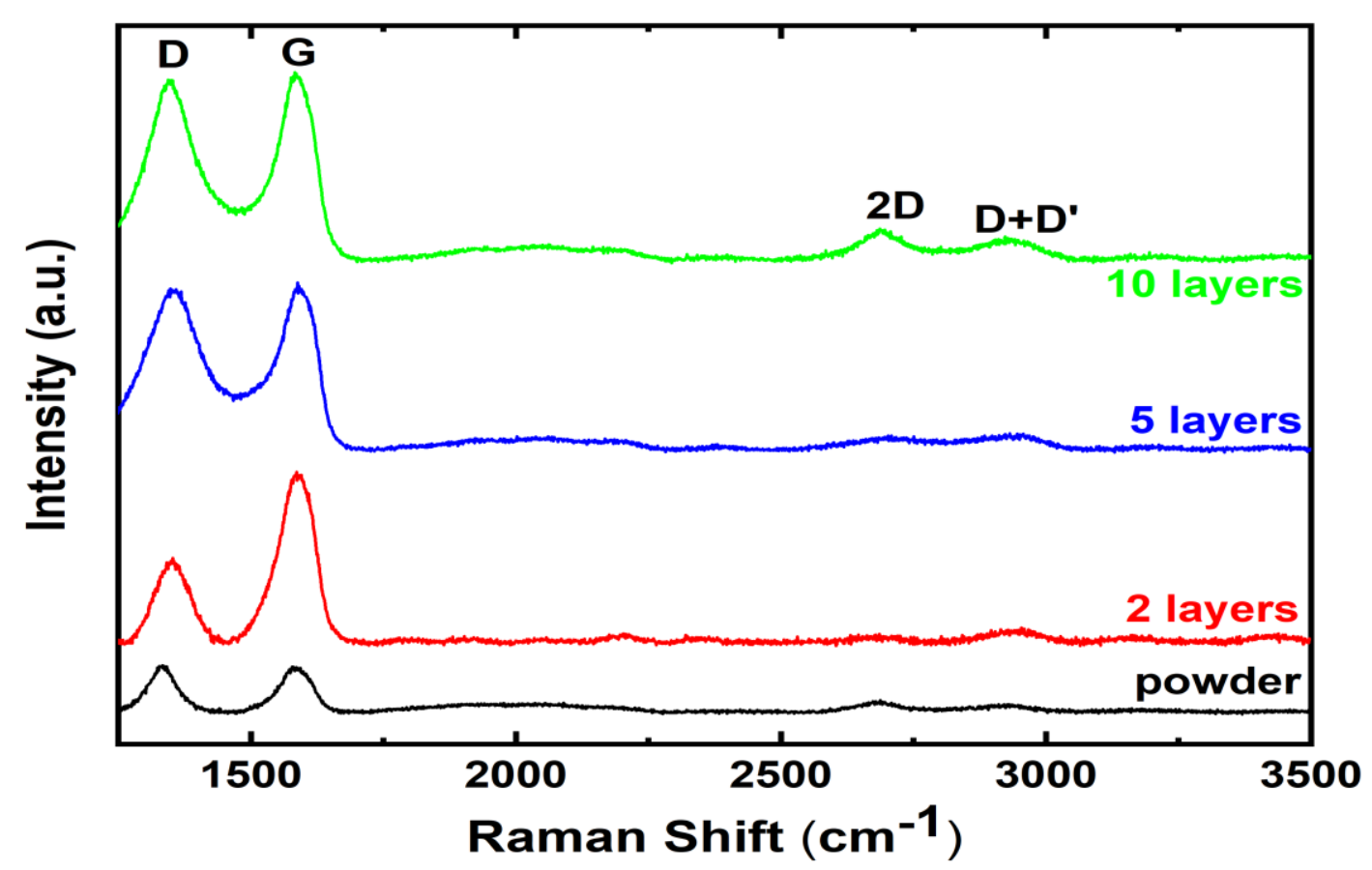

Figure 4. Raman spectra of printed NDG flakes compared with NDG powder.

\section{3. $\mathrm{MoS}_{2}-\mathrm{NDG}$ Stack}

The 2D materials can be arranged into a stack to create a materials assembly with novel properties [23]. In the stack, atomically thin 2D materials are bonded by weak van der Walls forces [24]. The $\mathrm{MoS}_{2}$ dispersions produced by LPE have the optical bandgap ranges from $1.8 \mathrm{eV}$ to $1.3 \mathrm{eV} \mathrm{[12].}$ We created and optimized the stack structures of printed $\mathrm{MoS}_{2}$ and NDG materials. To inspect the inside of a stack, a cross-sectional slice was produced by focused ion beam (FIB) milling for a thicker sample. Figure 5a shows the FIB-SEM cross-sectional image of the $\mathrm{MoS}_{2}-\mathrm{NDG}$ stack. The cross-sectional thickness of the NDG and $\mathrm{MoS}_{2}$ prints are 527 and $105 \mathrm{~nm}$, respectively. The interfacial separation between the $\mathrm{MoS}_{2}$ and NDG is not abrupt and well-maintained along the cross-section. There is no identifiable large flake in the cross-section. This ensures that the nanosheets are dissolved well in the solvents, and our sonication and centrifugation processes are reliable in removing thicker flakes. Raman of the $\mathrm{MoS}_{2}-\mathrm{NDG}$ stack in Figure $5 \mathrm{~b}$ shows the characteristic $\mathrm{MoS}_{2}$ peaks $\left(\mathrm{E}_{2 \mathrm{~g}} \sim 378 \mathrm{~cm}^{-1}\right.$, $\mathrm{A}_{1 \mathrm{~g}} \sim 403 \mathrm{~cm}^{-1}$ ) and NDG peaks ( $\sim 1357 \mathrm{~cm}^{-1}, \mathrm{G} \sim 1590 \mathrm{~cm}^{-1}$, and $2 \mathrm{D} \sim 2681 \mathrm{~cm}^{-1}$ ). 

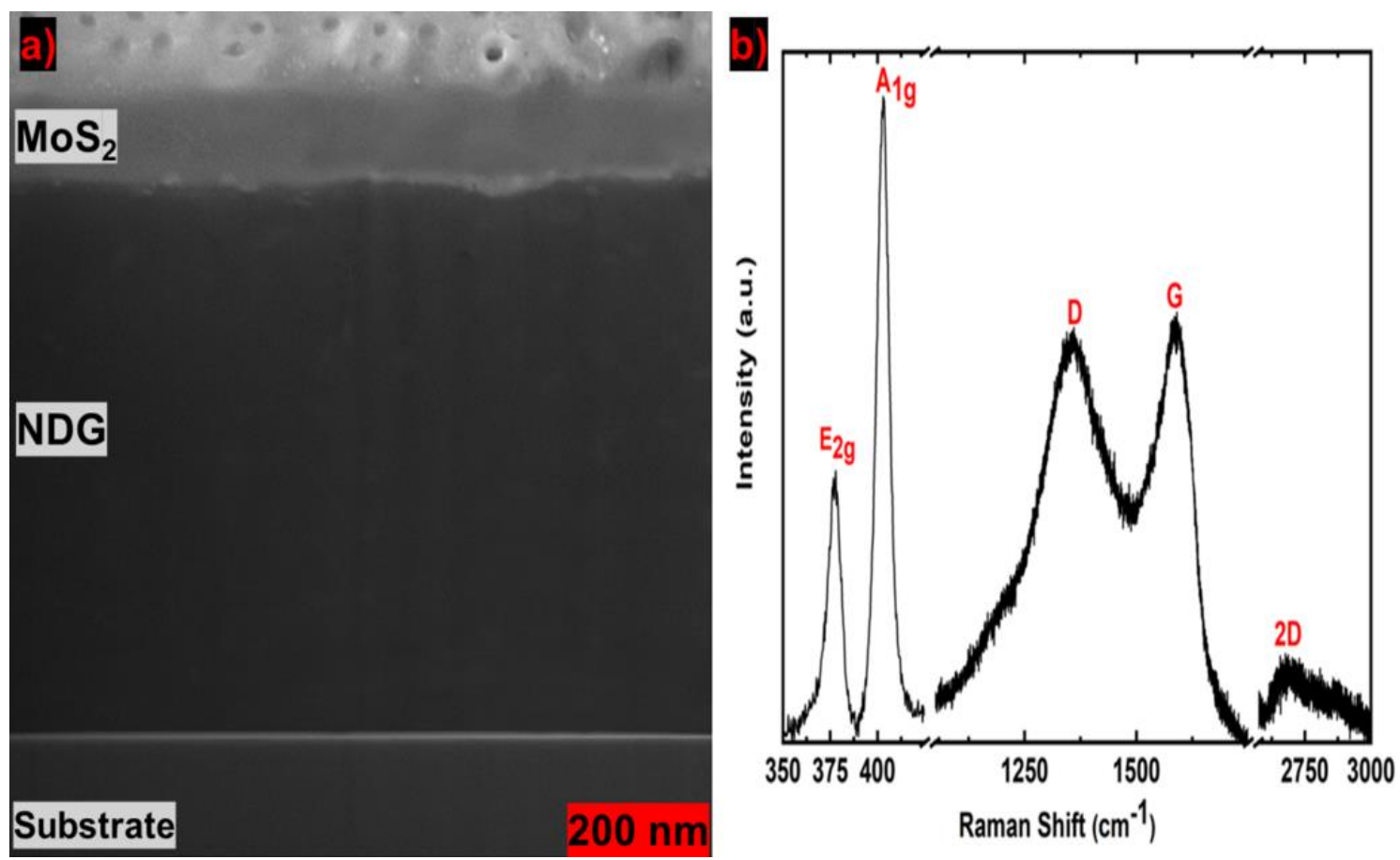

Figure 5. (a) FIB-SEM cross-sectional image of the $\mathrm{MoS}_{2}-\mathrm{NDG}$ stack, and (b) Raman spectrum of the printed $\mathrm{MoS}_{2}-\mathrm{NDG}$ stack on a glass substrate.

\subsection{Transistor Fabrication and Characterization}

The transistors themselves are all inkjet printed on the glass substrate without involving any photolithography patterning or surface pretreatment steps. The TFTs are top gated, consisting of the source, drain, gate electrodes, a channel, and a dielectric layer. The inset of Figure 6a shows the schematic structure of an NDG transistor. Highly conductive silver nanoparticle ink (Novacentrix Metalon ${ }^{\circledR}$ JS-B40G) was used to print source, drain and gate terminals. As the very first layer, source and drain electrodes were printed on the glass substrate in a printing pass. The printed silver patterns were cured at $150{ }^{\circ} \mathrm{C}$ for $30 \mathrm{~min}$. The separation between source and drain electrodes, i.e., the channel length (L) was $\sim 80 \mu \mathrm{m}$. The ed silver contacts deposited uniformly with an average thickness of $0.50 \mu \mathrm{m}$. For channel region, a percolation network of $100 \mathrm{~nm}$ NDG was printed with 40 printing passes followed by a $\mathrm{MoS}_{2}$ printing repeated 4 times on top of NDG. The channel layer had some overlapping with source and drain contacts to avoid discontinuity in the structure. Barium titanate $\left(\mathrm{BaTiO}_{3}\right)$ ink $(k \sim 20.5)$ of a thickness of $\sim 2 \mu \mathrm{m}$ was used as the dielectric (see 'Materials and Methods'). The printed dielectric was first baked at $100^{\circ} \mathrm{C}$ for $20 \mathrm{~min}$, and subsequently baked at $230^{\circ} \mathrm{C}$ for $30 \mathrm{~min}$. Finally, the silver gate electrode was printed on top of the dielectric layer. 

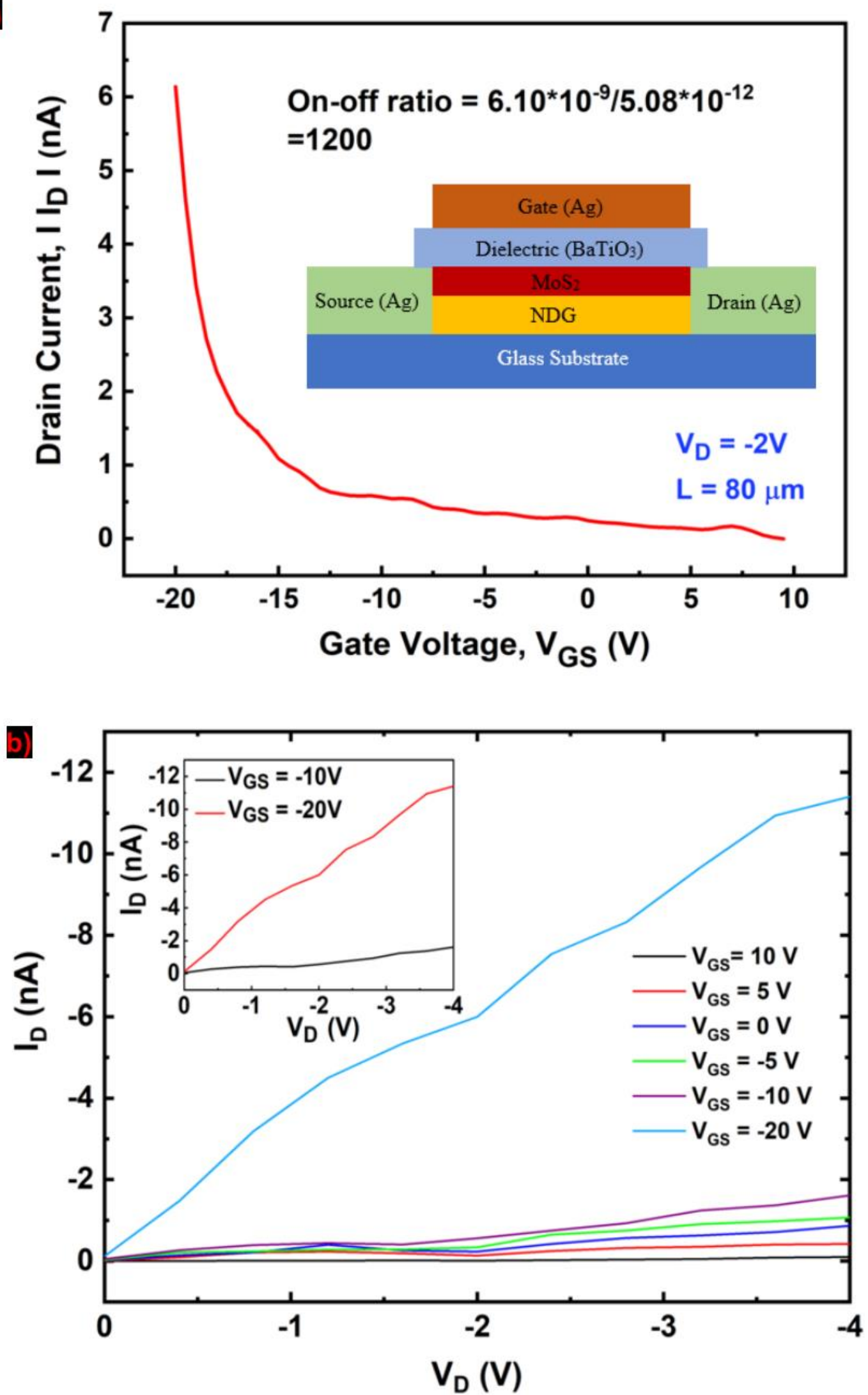

Figure 6. (a) Transfer curve of the $\mathrm{MoS}_{2}-\mathrm{NDG}$ transistor shown in the inset, and (b) output characteristics of the same device. 
Figure 6a shows the non-linear transfer curve of the transistor at $\mathrm{V}_{\mathrm{D}}=-2 \mathrm{~V}$. The applied gate voltage changed from -20 to $10 \mathrm{~V}$. The $\mathrm{I}_{\mathrm{D}}$ increases as the gate voltage changes from positive to negative, which suggests that the stack creates a p-type transport layer. A current on/off ratio of 1200 is calculated at $\mathrm{V}_{\mathrm{D}}=-2 \mathrm{~V}$ from the transfer characteristics. The electric field mobility $\left(\mu=\frac{\mathrm{L} \times \mathrm{g}_{\mathrm{m}}}{{\mathrm{W} \times \mathrm{C}_{\mathrm{ox}} \times \mathrm{V}_{\mathrm{D}}}_{\mathrm{D}}}\right)$ is calculated under the assumption of constant electric field along the channel and no velocity saturation. The device mobility was $\sim 0.01 \mathrm{~cm}^{2} / \mathrm{V} . \mathrm{s}$, which is comparable with liquid-exfoliated $\mathrm{MoS}_{2}-$ based TFTs [8]. Moreover, the device has a good off state. We measured the gate leakage $\left(\mathrm{I}_{\mathrm{G}}\right)$ from -10 to $+10 \mathrm{~V}$. The $\mathrm{I}_{\mathrm{G}}$ shows random signal in this range and does not indicate any gate leakage and the drain current $\left(\mathrm{I}_{\mathrm{D}}\right)$ was independent of the gate current (Supplementary Information). Figure 6b shows the output characteristics of the device at a gate voltage sweep from 10 to $-20 \mathrm{~V}$. There is no Schottky barrier and the current started flowing upon applying $\mathrm{V}_{\mathrm{D}}$ implying the contact is ohmic and is the result of direct tunneling process. The transfer and output characteristics of the device exhibit p-type behaviors. To benchmark the on/off ratio results, we also investigated the inkjet-printed NDG transistors. The similar fabrication procedures were carried out for NDG transistors $(\mathrm{L} \sim 80 \mu \mathrm{m})$ on the glass substrate with same $\mathrm{Ag}$ electrodes and $\mathrm{BaTiO}_{3}$ gate dielectric. For NDG transistors, a channel of $100 \mathrm{~nm}$ thickness was created with 40 printing repetitions to ensure the availability of NDG nanosheets on the top surface of the NDG thin films. The channel layer had some overlapping with source and drain contacts to avoid discontinuity in the structure. Transfer characteristics of the NDG transistor are shown in Figure S4a. The gate voltages were changed from -20 to $+20 \mathrm{~V}$. The transfer characteristics were measured at $\mathrm{V}_{\mathrm{D}}=5,10,15$, and $20 \mathrm{~V}$, respectively. The drain current increased as the $V_{G S}$ changed from negative to positive, which confirmed the n-type doping in NDG nanosheets due to nitrogen doping. However, the drain current did not change significantly over a wide range of gate bias. For example, at $\mathrm{V}_{\mathrm{D}}=20 \mathrm{~V}$, the drain current changed from 1105 to $1247 \mathrm{~mA}$ in the given gate voltage range. The current on/off ratio can be calculated from the transfer characteristics and it is the ratio of maximum to minimum drain current for a specific $V_{D}$. At $V_{D}=20$, the on/off ratio of the NDG is $\sim 1.13$. This on/off ratio is comparable to other inkjet-printed graphene TFTs, but not suitable for transistor switches. The drain currents were measured in $\mathrm{V}_{\mathrm{GS}}=-20,-10,0,10$, and $20 \mathrm{~V}$, respectively. Still the linear $I_{D}-V_{D}$ curves were not distinguishable for various gate voltages, i.e., the field effect in the output curves were not apparent. A linear $I_{D}-V_{D}$ curve for $V_{G S}=10 \mathrm{~V}$ is displayed in Figure S4b as the output characteristics. We also fabricated several $\mathrm{MoS}_{2}$ transistors following same fabrication processes for a channel length of $\sim 80 \mu \mathrm{m}$. However, we were unsuccessful in achieving a working transistor. Our interpretation in this matter is that the printed $\mathrm{MoS}_{2}$ layers failed to form a semiconducting percolation network on a bare glass substrate. However, $\mathrm{MoS}_{2}$ layers when printed on top of NDG layers, formed a continuous $\mathrm{MoS}_{2}$ percolation network and maintained a clear separation from the NDG layers underneath due to hydrophobic nature of NDG surface. Some $\mathrm{MoS}_{2}$ flakes may penetrate to the NDG layers, which reduced the overall conductivity of the NDG channel. Therefore, the high current on/off ratio originated from printed $\mathrm{MoS}_{2}$ and the NDG layers underneath supported $\mathrm{MoS}_{2}$ to start the nucleation when printed.

\section{Discussion}

We demonstrated a high current on/off ratio transistor based on the $\mathrm{MoS}_{2}-\mathrm{NDG}$ stack as the channel. The inks, thin films, and devices were characterized by various experimental characterization techniques. The NDG transistor was used as a control structure which showed low on/off ratios. Upon printing $\mathrm{MoS}_{2}$ on top of NDG to create a $\mathrm{MoS}_{2}-\mathrm{NDG}$ stack, semiconducting properties were achieved, and on/off ratios increased significantly for a channel length of $80 \mu \mathrm{m}$.

\section{Materials and Methods}

Nitrogen-doped graphene (NDG) ink formulation. The NDG ink was prepared by dispersing NDG flakes into terpineol/cyclohexanone mixture. The NDG powder $(\approx 5 \mathrm{mg}$, Sigma-Aldrich product no. 900527, Saint Louis, MO 63103, USA) was sonicated for $10 \mathrm{~h}$ to break the bigger particles. For a 
$10 \mathrm{~mL}$ solution, $1.5 \mathrm{~mL}$ of terpineol was used as the solvent. To curtail the viscosity of terpineol, 8.5 $\mathrm{mL}$ of cyclohexanone was added. Then, ethyl cellulose (as a surfactant) of $48 \mathrm{mg}$ was mixed into the solution and sonicated for $1 \mathrm{~h}$. Finally, the sonicated NDG powder $(\approx 5 \mathrm{mg})$ was poured into the solution. The resulting suspension was further sonicated for $20 \mathrm{~h}$, and then centrifuged at $5000 \mathrm{rpm}$ for $30 \mathrm{~min}$ to remove the thicker flakes. After centrifugation, the suspension was left to settle overnight. The final ink was filtered out from the residue and ready for characterization.

Nitrogen-doped graphene (NDG) ink characterization. The surface tension of NDG ink was measured using the pendant drop method (First Ten Angstroms FTA10000B, Portsmouth, VA 23704, USA). A small amount of ink $(3.8 \mu \mathrm{L})$ was suspended from a needle and surface tension was extracted from the software image. The ink density is $\rho=\mathrm{m} / \mathrm{V}, \mathrm{m}$ is the mass of ink in grams and $\mathrm{V}$ is volume of ink in $\mathrm{mL}$. The ink density $\rho$ for NDG ink was $1.054 \mathrm{~g} / \mathrm{cm}^{3}$. The viscosity of the ink was measured with the RheoSense $\mathrm{m}-\mathrm{VROC}$ viscometer. The viscosity of NDG ink was $\approx 2.94 \mathrm{mPa}$. Similar characterization was carried out for the $\mathrm{MoS}_{2}$ ink.

Atomic force microscopy (AFM). A Bruker atomic force microscope (Billerica, MA 01821, USA) was used to extract the flake thickness and lateral distribution. The flakes were scraped on a silicon substrate after sonication before mixing with solvents. The scan areas were $500 \times 500 \mu \mathrm{m}^{2}$ and $200 \times 200 \mu \mathrm{m}^{2}$ for the nanosheets.

Scanning electron microscopy. Scanning electron microscopy was carried out using a Helios Nanolab 400 FEI SEM (Hillsboro, OR 97124, USA). The field emission gun was operated at an accelerating voltage of $5 \mathrm{kV}$ and gun current of $0.34 \mathrm{nA}$. The images were captured in the field-free mode of SEM using TLD detector at the indicated magnification scale in SEM images.

Raman spectroscopy. Raman measurements were performed on the sonicated bulk NDG powder and inkjet-printed layers using a Horiba LabRAM HR evolution Raman spectrometer (Austin, TX 78754, USA). The laser excitation of $532 \mathrm{~nm}$ was used at $100 \times$ objective, with an incident power less than $1 \mathrm{~mW}$ to avoid sample damage.

Molybdenum disulfide $\left(\mathbf{M o S}_{2}\right)$ ink. The $\mathrm{MoS}_{2}$ ultrafine powder was purchased from Graphene Supermarket (Ronkonkoma, NY 11779, USA). The NMP (1-Methyl-2-pyrrolidinone) solvent was diluted to DI water by a 45:55 mL ratio. The weight of NMP/DI water composition was measured and mixed with $2 \%$ weight equivalent of $\mathrm{MoS}_{2}$ powder. The mixture was sonicated for $20 \mathrm{~h}$ and followed by a centrifugation at $5000 \mathrm{rpm}$ for $30 \mathrm{~min}$. The supernatant was filtered out from the top of the mixture after it rested overnight. The resultant $\mathrm{MoS}_{2}$ ink was yellowish. The ink viscosity, surface tension, and density were $3.8 \mathrm{mPa} \mathrm{s}, 25 \mathrm{mN} / \mathrm{m}$, and $0.1 \mathrm{~g} / \mathrm{cm}^{3}$, respectively.

Optical absorption spectroscopy. UV-vis absorption spectroscopy was used to calculate the flakes concentration in NDG and $\mathrm{MoS}_{2}$ inks utilizing the Beer-Lambert law. For the absorbance spectra measurements, the NDG ink was diluted in isopropyl alcohol (IPA) at a ratio of 1:20 and the $\mathrm{MoS}_{2}$ ink was as prepared. The measurements were performed with a Shimadzu UV-2501 spectrophotometer (Kyoto, Japan) using a glass cuvette with an optical path length of $10 \mathrm{~mm}$. For concentration calculation, absorption coefficients of $\alpha_{\mathrm{GR}} \sim 2460$ for the graphene ink at $660 \mathrm{~nm}$ wavelength [21], and $\alpha_{\mathrm{MoS2}} \sim 3400$ for the $\mathrm{MoS}_{2}$ ink [8] at $672 \mathrm{~nm}$ wavelength were used.

Barium titanate $\left(\mathrm{BaTiO}_{3}\right)$ ink preparation. The dielectric ink includes $20 \%$ by weight of $\mathrm{BaTiO}_{3}$ nanoparticles, $1 \%$ by weight of the Triton X-100, 14.5\% by weight of Cyclopentanone, and $64.5 \%$ by weight of SU8 2005 photoresist. For ink preparation, SU8 2005 (MicroChem, Newton, MA 02464, USA), Cyclopen-tanone (Sigma-Aldrich, product-W391018), and Triton X-100 (Sigma-Aldrich, product-X100) were mixed and sonicated for $4 \mathrm{~h}$. The $\mathrm{BaTiO}_{3}$ nanoparticles (US Research Nanomaterials, product-US3827, Houston, TX 77084, USA) are then added to the mixture, and the composition was sonicated for an additional $6 \mathrm{~h}$. The resultant mixture was left untouched for one day to form a stable dispersion. The ink was collected from the suspension, leaving the residue of thick powders at the bottom of the bottle. The density of $\mathrm{BaTiO}_{3}$ was measured to be $1.4 \mathrm{~g} / \mathrm{cm}^{3}$. The surface tension of $55 \mathrm{mN} / \mathrm{m}$ and viscosity of $12 \mathrm{mPa}$ s were considered for inverse Ohnesorge number calculation [25]. The effective dielectric constant $(k)$ of the printed dielectric was measured 
from the transmission coefficient $\mathrm{S}_{12}$ with a T-shape microstrip line resonator. The effective dielectric constant $(k)$ was $\sim 20.5$. The $k$-value of printed dielectric is consistent with the previous report [26].

Viscosity measurement. The viscosity was measured at room temperature with a Rheosense m-VROC viscometer (San Ramon, CA 94583, USA). In m-VROC Rheosense software (version: 3.1.4), we entered the sample size in $\mathrm{mL}$ and an estimated viscosity for an ink and it automatically calculated minimum and maximum flow rate. We set five different flow rates within the displayed range and measurement times and dispersed the sample through the measurement sensor. The regression coefficient $\left(R^{2}\right)$ was greater than 0.99 for all our viscosity measurements

Inkjet Printing. A DoD Fujifilm Dimatix (DMP-2800, Santa Clara, CA 95050, USA) inkjet printer was used to print all the thin films and devices. It was equipped with a Dimatix materials cartridge (DMC-11610). The cartridge head has 16 piezoelectric jetting nozzles with a diameter of $21.5 \mu \mathrm{m}$ each. The nozzles can dispense a droplet of the nominal volume of $10 \mathrm{pL}$ from the cartridge head. The optimum drop spacing for the NDG and MoS2 inks was $30 \mu \mathrm{m}$, and six nozzles were used to print these inks. The $\mathrm{BaTiO}_{3}$ ink was printed with a $35 \mu \mathrm{m}$ drop spacing with one nozzle. There was no delay between subsequent printing passes except a $0.3 \mathrm{~s}$ purge in every ten printing passes. Additionally, to avoid the coffee ring effect, the platen temperature was set at $60^{\circ} \mathrm{C}$. The $2 \mathrm{D}$ device patterns were created in AutoCAD, where the design layout represents different parts of the transistor. The design patterns were converted into a printable version of the Fujifilm Dimatix printer with ACE 300 software.

Electrical characterization. The current-voltage measurements of the devices were performed with the Keysight B1500A semiconductor device analyzer.

\section{Summary}

In summary, we developed the NDG and $\mathrm{MoS}_{2}$ inks for inkjet printing and studied the inks and thin-film properties through experimental characterizations. We devised a way to prepare a $\mathrm{MoS}_{2}-\mathrm{NDG}$ stack to use as the transistor channel. The devices were fully inkjet-printed without any involvement of Cleanroom facility. Based on the test results of the DC analysis of the device, the current on/off ratio of 1200 is reported in this work.

Supplementary Materials: The following are available online. Table S1: Data table for Raman measurements; Figure S1: Optical image of final transistor; Figure S2: Absorbance spectrum for solvents of (a) NDG ink, and (b) $\mathrm{MoS}_{2}$ ink; Figure S3: Gate leakage measurement of the $\mathrm{MoS}_{2}-\mathrm{NDG}$ transistor; Figure S4: (a) Transfer characteristics, and (b) output curve of the NDG transistor; Table S2: Comparison of on/off ratios of 2D material TFTs; Figure S5: Comparison of current on/off ratios.

Author Contributions: M.U.J. designed the experiments, developed the inks, and printed TFTs; performed SEM, Raman characterization, UV-vis spectroscopy, and electrical measurements of the transistors; analyzed the results; wrote the manuscript. M.A.M. developed the nanosheet SEM and cross-sectional SEM images. B.M. performed AFM measurements of the NDG and $\mathrm{MoS}_{2}$ nanosheets. M.Y.C. supervised the whole project. All authors have read and agreed to the published version of the manuscript.

Funding: This work was supported by the grant from NASA STTR Phase II under contract no. NNX15CC34C, and Thesis Support Fellowship at Texas State University, San Marcos, Texas.

Conflicts of Interest: The authors declare no conflict of interest.

\section{References}

1. Geim, A.K.; Novoselov, K.S. The rise of graphene. Nat. Mater. 2007, 6, 183-191. [CrossRef] [PubMed]

2. Li, J.; Ye, F.; Vaziri, S.; Muhammed, M.; Lemme, M.C.; Östling, M. Efficient inkjet printing of graphene. Adv. Mater. 2013, 25, 3985-3992. [CrossRef] [PubMed]

3. Xia, F.; Farmer, D.B.; Lin, Y.; Avouris, P. Graphene field-effect transistors with high on/off current ratio and large transport band gap at room temperature. Nano Lett. 2010, 10, 715-718. [CrossRef] [PubMed]

4. Schwierz, F. Graphene transistors. Nat. Nanotechnol. 2010, 5, 487-496. [CrossRef]

5. Torrisi, F.; Hasan, T.; Wu, W.; Sun, Z.; Lombardo, A.; Kulmala, T.S.; Hsieh, G.-W.; Jung, S.; Bonaccorso, F.; Paul, P.J. Inkjet-printed graphene electronics. ACS Nano 2012, 6, 2992-3006. [CrossRef] 
6. Carey, T.; Cacovich, S.; Divitini, G.; Ren, J.; Mansouri, A.; Kim, J.M.; Wang, C.; Ducati, C.; Sordan, R.; Torrisi, F. Fully inkjet-printed two-dimensional material field-effect heterojunctions for wearable and textile electronics. Nat. Commun. 2017, 8, 1202. [CrossRef]

7. Li, X.; Zhu, H. Two-dimensional $\mathrm{MoS}_{2}$ : Properties, preparation, and applications. J. Mater. 2015, 1, 33-44. [CrossRef]

8. Coleman, J.N.; Lotya, M.; O’Neill, A.; Bergin, S.D.; King, P.J.; Khan, U.; Young, K.; Gaucher, A.; De, S.; Smith, R.J. Two-dimensional nanosheets produced by liquid exfoliation of layered materials. Science 2011, 331, 568-571. [CrossRef]

9. Lee, K.; Kim, H.; Lotya, M.; Coleman, J.N.; Kim, G.; Duesberg, G.S. Electrical characteristics of molybdenum disulfide flakes produced by liquid exfoliation. Adv. Mater. 2011, 23, 4178-4182. [CrossRef]

10. Li, J.; Naiini, M.M.; Vaziri, S.; Lemme, M.C.; Östling, M. Inkjet printing of $\mathrm{MoS}_{2}$. Adv. Funct. Mater. 2014, 24, 6524-6531. [CrossRef]

11. He, Q.; Zeng, Z.; Yin, Z.; Li, H.; Wu, S.; Huang, X.; Zhang, H. Fabrication of flexible $\mathrm{MoS}_{2}$ thin-film transistor arrays for practical gas-sensing applications. Small 2012, 8, 2994-2999. [CrossRef]

12. Kelly, A.G.; Hallam, T.; Backes, C.; Harvey, A.; Esmaeily, A.S.; Godwin, I.; Coelho, J.; Nicolosi, V.; Lauth, J.; Kulkarni, A. All-printed thin-film transistors from networks of liquid-exfoliated nanosheets. Science 2017, 356, 69-73. [CrossRef]

13. Lin, Z.; Liu, Y.; Halim, U.; Ding, M.; Liu, Y.; Wang, Y.; Jia, C.; Chen, P.; Duan, X.; Wang, C. Solution-processable 2D semiconductors for high-performance large-area electronics. Nature 2018, 562, 254-258. [CrossRef] [PubMed]

14. Zhou, S.Y.; Gweon, G.-H.; Fedorov, A.V.; First de, P.N.; De Heer, W.A.; Lee, D.-H.; Guinea, F.; Neto, A.H.C.; Lanzara, A. Substrate-induced bandgap opening in epitaxial graphene. Nat. Mater. 2007, 6, 770-775. [CrossRef] [PubMed]

15. Lawlor, J.A.; Ferreira, M.S. Sublattice asymmetry of impurity doping in graphene: A review. Beilstein J. Nanotechnol. 2014, 5, 1210-1217. [CrossRef] [PubMed]

16. Han, M.Y.; Özyilmaz, B.; Zhang, Y.; Kim, P. Energy bandgap engineering of graphene nanoribbons. Phys. Rev. Lett. 2007, 98, 206805. [CrossRef]

17. Koh, Y.K.; Bae, M.-H.; Cahill, D.G.; Pop, E. Reliably counting atomic planes of few-layer graphene $(\mathrm{n}>4)$. ACS Nano 2010, 5, 269-274. [CrossRef]

18. Addou, R.; Colombo, L.; Wallace, R.M. Surface defects on natural MoS 2 . ACS Appl. Mater. Interfaces 2015, 7, 11921-11929. [CrossRef]

19. Ferrari, A.C.; Meyer, J.C.; Scardaci, V.; Casiraghi, C.; Lazzeri, M.; Mauri, F.; Piscanec, S.; Jiang, D.; Novoselov, K.S.; Roth, S. Raman spectrum of graphene and graphene layers. Phys. Rev. Lett. 2006, 97, 187401. [CrossRef]

20. Lu, Y.-F.; Lo, S.-T.; Lin, J.-C.; Zhang, W.; Lu, J.-Y.; Liu, F.-H.; Tseng, C.-M.; Lee, Y.-H.; Liang, C.-T.; Li, L.-J. Nitrogen-doped graphene sheets grown by chemical vapor deposition: Synthesis and influence of nitrogen impurities on carrier transport. ACS Nano 2013, 7, 6522-6532. [CrossRef]

21. Hernandez, Y.; Nicolosi, V.; Lotya, M.; Blighe, F.M.; Sun, Z.; De, S.; McGovern, I.T.; Holland, B.; Byrne, M.; Gun'Ko, Y.K. High-yield production of graphene by liquid-phase exfoliation of graphite. Nat. Nanotechnol. 2008, 3, 563-568. [CrossRef] [PubMed]

22. Mishra, A.K.; Lakshmi, K.V.; Huang, L. Eco-friendly synthesis of metal dichalcogenides nanosheets and their environmental remediation potential driven by visible. Sci. Rep. 2015, 5, 15718. [CrossRef] [PubMed]

23. Bonaccorso, F. Ink-jet printed 2D crystal heterostructures. In Proceedings of the 2017 47th European Solid-State Device Research Conference (ESSDERC), Leuven, Belgium, 11-14 September 2017; Institute of Electrical and Electronics Engineers (IEEE): Los Alamitos, CA, USA; pp. 208-211. [CrossRef]

24. Roy, T.; Tosun, M.; Kang, J.S.; Sachid, A.B.; Desai, S.B.; Hettick, M.; Hu, C.C.; Javey, A. Field-effect transistors built from all two-dimensional material components. ACS Nano 2014, 8, 6259-6264. [CrossRef] [PubMed]

25. Vukmirovic, J.; Tripkovic, D.; Bajac, B.; Kojic, S.; Stojanovic, G.; Srdic, V.V. omparison of barium titanate thin films prepared by inkjet printing and spin coating. Process. Appl. Ceram. 2015, 9, 151-156. [CrossRef] 
26. Lau, P.H.; Takei, K.; Wang, C.; Ju, Y.; Kim, J.; Yu, Z.; Takahashi, T.; Cho, G.; Javey, A. Fully printed, high performance carbon nanotube thin-film transistors on flexible substrates. Nano Lett. 2013, 13, 3864-3869. [CrossRef] [PubMed]

Sample Availability: Samples are not available from the authors.

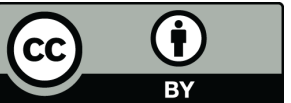

(C) 2020 by the authors. Licensee MDPI, Basel, Switzerland. This article is an open access article distributed under the terms and conditions of the Creative Commons Attribution (CC BY) license (http://creativecommons.org/licenses/by/4.0/). 